\title{
Metastasis Directed Therapy and/or Systemic Therapy in Hormone-Naive Oligometastatic Prostate Cancer Patient: an Emerging Dilemma
}

\author{
Francesco Cuccia $^{1}$ and Filippo Alongi ${ }^{1,2}$ \\ ${ }^{1}$ Advanced Radiation Oncology Department - Sacro Cuore Don Calabria Hospital, Negrar di \\ Valpolicella (VR), Italy \\ ${ }^{2}$ University of Brescia, Brescia, Italy
}

Corresponding author: Francesco Cuccia e-mail: francesco.cuccia@sacrocuore.it

\begin{abstract}
The hormone-naive oligometastatic prostate cancer is a challenging setting for the radiation oncology community, as it represents a sort of transition scenario potentially suitable for two different approaches: a local ablative treatment alone vs a metastasisdirected treatment with the addition of hormone therapy. The choice to add androgen deprivation therapy in the oligorecurrent hormone-sensitive patient is a matter of debate, given the detrimental impact on quality of life and the number of adverse events. To date, there is no clear agreement on the optimal management of this subset of patients. As some authors highlight the attractiveness of a local approach alone, as well tolerated, easily repeatible and with very limited costs, on the other hand, other authors focus the need to cover the micrometastatic disease, often not detectable, even with the newly available imaging modalities. In this commentary, we briefly summarize the literature data in support of both therapeutic strategies.
\end{abstract}

Keywords: Prostate cancer, Oligometastatic cancer, SBRT, Androgen Deprivation Therapy, Radiotherapy

\section{Main text}

Since 1995 with the birth of the concept of the oligometastatic disease, as defined by Hellman and Weichselbaum, the spectrum of metastatic tumors has been revised, depending on the timing of onset (synchronous vs metachronous), or based on the presence/absence of ongoing systemic treatments (oligorecurrent vs oligoprogressive vs oligopersistent metastases).

In the setting of the oligometastatic disease, in the last years, a large amount of 
literature studies have supported the beneficial impact of the so-called metastasisdirected therapies (MDT) as a means to gain an advantage in terms of clinical outcomes [1,2].

Specifically for oligometastatic prostate cancer, the administration of stereotactic body radiotherapy (SBRT) has been favorably reported as a safe and effective tool, not only in terms of direct local control, but also as an option to delay the natural history of the disease by postponing the start of systemic therapies [3-6].

Infact, a major concern for this subset of patients is represented by the use of androgen deprivation therapy (ADT), since many patients would avoid the adverse effects and the detrimental impact of hormone therapy in terms of quality of life. In this scenario, the global widespread of metastasis-directed therapy is strictly connected to the availability of new diagnostic tools, such as multiparametric magnetic resonance or newer metabolic tracers for PET-imaging, which have significantly improved the sensitivity and specificity for the identification of the realoligometastatic patient, who may really benefit from a local approach [7].

The role of newer metabolic tracers for the patient's selection process has been supported also by Fossati et al. [8] in a large multi-center series of patients which were candidate to salvage lymph-node dissection for nodal recurrence. The authors aimed to identify predictive factors of recurrence, in order to refine the accuracy for the identification of the best fit patient for directed surgical approach.

This has been recently confirmed by the randomized phase II trial ORIOLE, in which a statistically significant advantage provided by SBRT was detected in terms of progression-free survival, when compared to observation in the hormone-sensitive oligometastatic patient. Moreover, the authors reported better progression-free survival rates in patients who reached a complete metabolic response after 6 months. This evidence highlights the need to better focus the patient's selection process, since it is a crucial step in determining the best therapeutic strategy. As recorded by Phillips et al. in their study [9], an incomplete prostate specific antigen (PSA) or absence of metabolic response may suggest the persistence of micrometastatic disease, thus making MDT as a clock reset to the onset of detectable macrometastases. Still, also in this case, MDT remains an attractive option due to the possibility to repeat multiple rounds of local approaches, with no relevant side effects, until a poly-metastatic progression takes place [10]. Furthermore, some authors also hypothesize a SBRTinduced immune effect in affecting the signals promoting the development of the metastatic "iceberg", leading also to potentially overcome, in the next years, the numerical concept of oligometastatic disease in favor of a deeper knowledge of the biological features of the metastatic cells [11]. 
This has been recently suggested also by Deek et al. [12] in a retrospective study of 269 samples from metastatic castrationsensitive prostate cancer patients with the aim to characterize the biological features of the real oligometastatic disease. The authors focus the need to identify genomic signatures as prognostic factors helpful to delineate the perfect candidates for MDT consolidative approaches. Probably, the combination of the mutational features and of the diagnostic imaging modalities will increase the accuracy in the detection of the real metastatic burden allowing clinicians to perform "sensu stricto" ablative treatments potentially aiming to cure the patient [13].

Moreover, the use of MDT approaches may have a favorable impact from a socioeconomic point of view, given the possibility to delay the administration not only of conventional ADT drugs but also for second generation antiandrogens [14].

On the contrary, the early activation of ADT is a matter of debate, with some studies reporting an OS advantage compared to delayed strategies [15]. Also, the optimal management of ADT is controversial, although continuous ADT in the metastatic setting has been proved to be superior to intermittent administration in terms of clinical outcomes, still at the price of a worse impact on quality of life [16, 17].

Moreover, the logistics for intermittent ADT are controversial, especially for the criteria for stopping or resuming the treatment [18].
In a recent literature review by Carrasquilla et al., the authors hypothesize a risk-adapted intermittent ADT strategy for the management of lymph-node oligorecurrent hormonesensitive prostate cancer also including an involved field SBRT in order to ablate the potential castration-resistant clones, thus prolonging the efficacy of intermittent hormone therapy [19].

In a recent manuscript by Chaw et al. [20], a randomized phase II study measured the impact of immediate ADT + SBRT vs SBRT + delayed ADT in a cohort of 88 patients with up to 3 oligometastates. The authors observed better clinical outcomes in the arm with immediate ADT suggesting a potential synergistic effect of the combined treatment. However, major concerns remain both in terms of survival benefits, and adverse events due to long-term systemic therapy. Moreover, the delay of ADT may also delay the transition to the castrationresistant phase, as also supported by Studer et al [21].

Furthermore, specifically in the case of lymph-node oligometastases, the optimal management remains controversial, as in opposition to MDT, some authors also support elective nodal irradiation, being associated with a lower rate of recurrence, despite only retrospective studies to date are available, still reporting an increased incidence of toxicity compared to MDT [22].

Of note, lymph-node metastases are associated to a higher median OS as compared to other anatomic sites. As also highlighted by Tran et al. [23], probably the 
choice between a focal approach versus an elective treatment may be guided not only by appropriate imaging (i.e. Choline or PSMA$\mathrm{PET} / \mathrm{CT}$ ) but also by PSA doubling time (PSADT); longer PSADT may suggest a more indolent natural history of the disease, thus more suitable for focal and potentially curative approaches. Otherwise, in the case of shorter PSADT, an elective nodal irradiation may cover also the occult micrometastatic disease, which may not be detected even by the newer metabolic tracers. Of note, also the number of metastastic lymph-nodes is another parameter that must be taken into account for the choice of the best tailored approach [24].

As far as bone oligometastases, given the lack of prospective data, a wide range of variability remains between institutions, and the use of ADT remains a matter of debate, as some authors hypothesize the applicability of the same MDT strategy such as for lymph-nodal oligometastases [25]. Probably, also in this setting, the same indicators mentioned for lymph-nodes (PSADT, number of metastases) are helpful tools to guide clinicians in the choice of the best therapeutic management. Of course, the retrospective nature of the currently available evidence limits the power of our conclusions. Surely, stronger evidence concerning the role of MDT in metastatic prostate cancer will be provided by the ongoing randomized trials PEACE $V$ (NCT03569241), STEREO-OS (NCT031 43322), ADOPT (NCT04302454), RADIOSA (NCT03940235) and CORE (NCT02759 783) $[14,26]$.

\section{Conclusions}

The management of the hormone-naive oligometastatic prostate cancer patient represents a real challenge for the radiation oncologist. New imaging modalities are leading to increase the accuracy in detecting the real disease burden, aiming to refine the features of the true oligometastatic patient, potentially curable with ablative metastasisdirected treatments. Promising data are derived from the literature, to date, and in our opinion, in light of the current lack of robust evidence, the addition of immediate ADT must be guided by the assessment of other parameters such as PSA doubling time, number and sites of oligometastases, life expectancy, etc. Probably in the next years, the growing availability of more mature data and the deeper knowledge of the intrinsic biological features of the metastatic disease will provide further elements to help clinicians in the choice of the best tailored treatment.

\footnotetext{
Abbreviations:

ADT - androgen deprivation therapy

MDT - metastasis-directed therapies

PET - positron emission tomography

PSA - prostate specific antigen

PSADT - doubling time

PSMA - prostate specific membrane antigen

SBRT - stereotactic body radiotherapy
} 


\section{Statements:}

Authors' contributions: FC and FA eqully contributed for the manuscript

Consent for publication: As the corresponding author, I confirm that the manuscript has been read and approved for submission by all co-authors.

Conflict of interest: All authors declare having no competing interests associated with this publication.

Funding Sources: This research did not receive any specific grant from funding agencies in the public, commercial, or not-for-profit sector.

\section{References:}

1. Lehrer EJ, Singh R, Wang M, et al. Safety and Survival Rates Associated With Ablative Stereotactic Radiotherapy for Patients With Oligometastatic Cancer: A Systematic Review and Meta-analysis. JAMA Oncol. 2021;7(1):92-106. doi:10.1001/jamaoncol.2020.6146

2. Palma DA, Olson R, Harrow S, Gaede S, Louie AV, Haasbeek C, et al. Stereotactic Ablative Radiotherapy for the Comprehensive Treatment of Oligometastatic Cancers: Long-Term Results of the SABR-COMET Phase II Randomized Trial. J Clin Oncol. 2020 Sep 1;38(25):2830-2838. doi: 10.1200/JCO.20.00818.

3. Nicosia L, Franzese C, Mazzola R, et al. Recurrence pattern of stereotactic body radiotherapy in oligometastatic prostate cancer: a multi-institutional analysis. Strahlenther Onkol 2020; 196(3):213-21

4. Triggiani L, Alongi F, Buglione M, Detti B, Santoni R, Bruni A, et al. Efficacy of stereotactic body radiotherapy in oligorecurrent and in oligoprogressive prostate cancer: new evidence from a multicentric study. $\mathrm{Br} \mathrm{J} \mathrm{Cancer.} 2017$ Jun 6;116(12):1520-1525. doi: 10.1038/bjc.2017.103.

5. Mazzola R, Francolini G, Triggiani L, Napoli G, Cuccia F, Nicosia L, et al. Metastasis-directed Therapy (SBRT) Guided by PET-CT 18F-CHOLINE Versus PET-CT 68Ga-PSMA in Castration-sensitive Oligorecurrent Prostate Cancer: A Comparative Analysis of Effectiveness. Clin Genitourin Cancer. 2020 Aug 6:S15587673(20)30191-9. doi: 10.1016/j.clgc.2020.08.002. Epub ahead of print. PMID: 32863189.

6. Ost P, Reynders D, Decaestecker K, Fonteyne V, Lumen N, De Bruycker A, et al. Surveillance or MetastasisDirected Therapy for Oligometastatic Prostate Cancer Recurrence: A Prospective, Randomized, Multicenter Phase II Trial. J Clin Oncol. 2018 Feb 10;36(5):446-453. doi: 10.1200/JCO.2017.75.4853.

7. Mazzola R, Cuccia F, Figlia V, Giaj-Levra N, Nicosia L, Ricchetti F, et al. New metabolic tracers for detectable PSA levels in the post-prostatectomy setting: is the era of melting glaciers upcoming? Transl Androl Urol. 2019 Dec;8(Suppl 5):S538-S541. doi: 10.21037/tau.2019.12.34.

8. Fossati N, Suardi N, Gandaglia G, Bravi CA, Soligo M, Karnes RJ, et al. Identifying the Optimal Candidate for Salvage Lymph Node Dissection for Nodal Recurrence of Prostate Cancer: Results from a Large, Multi-institutional Analysis. Eur Urol. 2019 Jan;75(1):176-183. doi: 10.1016/j.eururo.2018.09.009.

9. Phillips R, Shi WY, Deek M, Radwan N, Lim SJ, Antonarakis ES, et al. Outcomes of Observation vs Stereotactic Ablative Radiation for Oligometastatic Prostate Cancer: The ORIOLE Phase 2 Randomized Clinical Trial. JAMA Oncol. 2020 May 1;6(5):650-659. doi: 10.1001/jamaoncol.2020.0147.

10. Greco C, Fuks Z. Forging New Strategies in the Cure of Human Oligometastatic Cancer. JAMA Oncol. 2020;6(5):659-660. doi:10.1001/jamaoncol.2020.0195

11. Greco C, Pares O, Pimentel N, Louro V, Morales J, Nunes B, et al. Phenotype-oriented ablation of oligometastatic cancer with single dose radiation therapy. Int J Radiat Oncol Biol Phys. 2019; $104(3): 593-603$. https://doi.org/10.1016/j.ijrob p.2019.02.033

12. Deek MP, Van der Eecken K, Phillips R, Parikh NR, Isaacsson Velho P, Lotan TL, et al. The Mutational Landscape of Metastatic Castration-sensitive Prostate Cancer: The Spectrum Theory Revisited. Eur Urol. 2021 Jan 5:S0302-2838(20)31026-5. doi: 10.1016/j.eururo.2020.12.040. 
13. Adson MA, van Heerden JA, Adson MH, Wagner JS, Ilstrup DM. Resection of hepatic metastases from colorectal cancer. Arch Surg 1984;119:647-51.

14. Marvaso G, Ciardo D, Corrao G, Gandini S, Fodor C, Zerini D, et al. Radioablation +/- hormonotherapy for prostate cancer oligorecurrences (Radiosa trial): potential of imaging and biology (AIRC IG-22159). BMC Cancer. 2019 Sep 10;19(1):903. doi: 10.1186/s12885-019-6117-z.

15. Duchesne GM, Woo HH, Bassett JK, Bowe SJ, D'Este C, Frydenberg M, et al.Timing of androgen-deprivation therapy in patients with prostate cancer with a rising PSA (TROG 03.06 and VCOG PR 01-03 (TOAD)): a randomised, multicentre, non-blinded, phase 3 trial. Lancet Oncol. 2016 Jun;17(6):727-737. doi: 10.1016/S1470-2045(16)001078.

16. Magnan S, Zarychanski R, Pilote L, et al. Intermittent vs Continuous Androgen Deprivation Therapy for Prostate Cancer: A Systematic Review and Meta-analysis. JAMA Oncol. 2015;1(9):1261-1269. doi:10.1001/jamaoncol.2015.2895

17. Calais da Silva F, Calais da Silva FM, Gonçalves F, Santos A, Kliment J, Whelan P, et al. Locally advanced and metastatic prostate cancer treated with intermittent androgen monotherapy or maximal androgen blockade: results from a randomised phase 3 study by the South European Uroncological Group. Eur Urol (2014) 66(2):232-9. doi: 10.1016/j.eururo.2013.03.055

18. Hussain M, Tangen CM, Higano C, Schelhammer PF, Faulkner J, Crawford ED, et al. Absolute prostatespecific antigen value after androgen deprivation is a strong independent predictor of survival in new metastatic prostate cancer: data from Southwest Oncology Group Trial 9346 (INT-0162). J Clin Oncol (2006) 24(24):3984-90. doi: $10.1200 / J C 0.2006 .06 .4246$

19. Carrasquilla M, Creswell ML, Pepin AN, Wang E, Forsthoefel M, McGunigal M, et al. Rationale for Involved Field Stereotactic Body Radiation Therapy-Enhanced Intermittent Androgen Deprivation Therapy in HormoneSensitive Nodal Oligo-Recurrent Prostate Cancer Following Prostate Stereotactic Body Radiation Therapy. Front Oncol. 2021 Jan 18;10:606260. doi: 10.3389/fonc.2020.606260.

20. Chaw CL, deSouza NM, Khoo V, Suh YE, van As N. Clinical Outcomes of Stereotactic Body Radiotherapy With Immediate Versus Delayed Hormone Therapy in Men With Oligometastatic Recurrence of Prostate Cancer. Clin Oncol (R Coll Radiol). 2020 Aug;32(8):509-517. doi: 10.1016/j.clon.2020.03.008.

21. Studer UE, Whelan P, Wimpissinger F, Casselman J, de Reijke TM, Knönagel H, et al; EORTC Genitourinary Cancer Group. Differences in time to disease progression do not predict for cancer-specific survival in patients receiving immediate or deferred androgen-deprivation therapy for prostate cancer: final results of EORTC randomized trial 30891 with 12 years of follow-up. Eur Urol. 2014 Nov;66(5):829-38. doi: 10.1016/j.eururo.2013.07.024.

22. De Bleser E, Jereczek-Fossa BA, Pasquier D, Zilli T, Van As N, Siva S, et al. Metastasis-directed Therapy in Treating Nodal Oligorecurrent Prostate Cancer: A Multi-institutional Analysis Comparing the Outcome and Toxicity of Stereotactic Body Radiotherapy and Elective Nodal Radiotherapy. Eur Urol. 2019 Dec;76(6):732-739. doi: 10.1016/j.eururo.2019.07.009.

23. Tran S, Jorcano S, Falco T, Lamanna G, Miralbell R, Zilli T. Oligorecurrent Nodal Prostate Cancer: Longterm Results of an Elective Nodal Irradiation Approach. Am J Clin Oncol. 2018 Oct;41(10):960-962. doi: 10.1097/COC.0000000000000419.

24. Crehange G, Roach M 3rd, Martin E, et al. Salvage reirradiation for locoregional failure after radiation therapy for prostate cancer: Who, when, where and how? Cancer Radiother. 2014;18:524-534.

25. Patel $\mathrm{PH}$, Chaw $\mathrm{CL}$, Tree AC, Sharabiani M, van As NJ. Stereotactic body radiotherapy for bone oligometastatic disease in prostate cancer. World J Urol. 2019 Dec;37(12):2615-2621. doi: 10.1007/s00345-01902873-w.

26. De Bruycker A, Spiessens A, Dirix P, Koutsouvelis N, Semac I, Liefhooghe N, et al. PEACE V - Salvage Treatment of OligoRecurrent nodal prostate cancer Metastases (STORM): a study protocol for a randomized controlled phase II trial. BMC Cancer. 2020 May 12; 20(1):406. doi: 10.1186/s12885-020-06911-4. 